[CONTRIBUTION FROM THE COMMITTEE ON LNIFORMITY IN TECHNICAL ANaLYSIS. IV.]

\title{
REPORT OF THE SUB-COMMITTEE ON SHELLAC ANALYSIS.
}

\author{
By Parker C. MCIlhiNey, A. C. IANgitir, Maximilias Toch, \\ Max Walzersters, acGCstes H. Gill, \\ Receired May 3 I, I907.
}

We have considered all the processes that have so far come to our 110tice having for their object the determination of rosin in mixtures of shellac with rosin, and find that several of the methods depending upon the different powers of shellac and of rosin to unite with the halogens are capable of being used to determine the proportions of rosin and shellac in their mixtures.

We have not found any of the methods in which use is made of the other properties of these two substances, such as solubility, the acidity, or the Köttstorfer figure, sufficiently reliable for general use.

As the absorption of halogen by both shellac and rosin is largely and perhaps entirely by substitution, and not as in the case of fats, by addition, and as the amount of halogen which so replaces hydrogen in the rosin is dependent upon the length of time that the reagent is allowed to act upon it, and also upon such other variables as the excess of the reagent, the temperature, and the relation of the volume of solution to the amount of substance treated, we have considered each of these halogen-absorption processes in the light of its adaptability to the maintenance of standard and uniform arbitrary conditions which will permit results obtained at one time being compared with those obtained at any other time.

The result of our work convinces us that the Wijs process ${ }^{1}$ used as proposed by Dr. Langmuir, one of our number, possesses the desirable features of regularity and reliability in a greater degree than any other process which has come to our notice. It is possible by it to carry out a test of a sample of shellac in a couple of hours, and as shellac is a rather expensive material and one very liable to sudden changes in price, it is almost essential that the standard method of testing it should be a rapid one. The Hiibl method is deficient in this respect, requiring usually 24 hours to carry out an analysis by it.

The method which we would recommend as a standard one for the determination of rosin in shellac is substantially the one described in Dr. Langmuir's paper in the J. Soc. Chem. Ind., 24, r2. The solutions required are one of iodine monochloride containing $\mathrm{I} 3 \mathrm{~g}$. of iodine per liter, in glacial acetic acid that has a melting point of $14.7^{\circ}$ to $15^{\circ} \mathrm{C}$. and is free from reducing impurities, and another of sodium thiosulphate made by dissolving $24.83 \mathrm{~g}$. of the pure salt in a liter of water. In addition to these solutions there is required a quantity of acetic acid of the same strength ${ }^{1}$ Ber.. 31, 750, and J. Soc. Chern. Ind., 17, 609. 
as that used for making the solution of iodine monochloride. Pure chloro form and starch are also necessary. The preparation of the iodine mono chloride solution presents no great difficulty, but it must be done with car and accuracy in order to obtain satisfactory results. There must be in th solution no sensible excess either of iodine or more particularly of chlo rine, over that required to form the monochloride. This condition $i$ most satisfactorily attained by dissolving in the whole of the acetic acic to be used the requisite quantity of iodine, using a gentle heat to assis the solution, if it is found necessary, setting asicle a small portion of thi solution, while pure and dry chlorine is passed into the remainder unti the halogen content of the whole solution is doubled. Ordinarily it wil be found that by passing the chlorine into the main part of the solution until the characteristic color of free iodine has just been discharged thers will be a slight excess of chlorine which is corrected by the addition 0 the requisite amount of the unchlorinated portion until all free chlorint has been destroyed. A slight excess of iodine does little or no harm, bu excess of chlorine must be avoided.

Introduce $0.200 \mathrm{~g}$. of ground shellac into a $250 \mathrm{cc}$. dry bottle 0 : clear glass with a ground glass stopper, add $20 \mathrm{cc}$. of glacial acetic acic and warm the mixture gently until solution is complett (except for the wax). A pure shellac is rather difficultly soluble. Solu. tion is quicker according to the proportion of rosin present; to cc. of chloroform are added and the solution is cooled to $2 \mathrm{I}^{\circ}$ to $24^{\circ}$. The temperature should be held well within these limits during the test. $20 \mathrm{cc}$. of Wijs solution are added from a pipette, haring a rather small delivery aperture. The bottle is closed and stood in a dark place, and the time noted. It is convenient to keep the bottles during the test partly immersed in water which should be kept as nearly as possible be. tween $22^{\circ}$ and $23^{\circ}$.

Pure shellac will scarcely alter the color of the Wijs solution. If in small amount, rosin will produce a slowly appearing red-brown color. In large amount, rosin causes an immediate coloration, increasing in intensity as time passes. After one hour, ro cc. of ro per cent. potassium iodide solution are added. The solution is immediately titrated with the sodium thiosulphate solution; $25 \mathrm{cc}$. or $30 \mathrm{cc}$. may be run in immediately, unless the shellac is very impure, and the remainder gradually with vigorous shaking. Just before the end, a little starch solution is added. The end point is sharp, as the reaction products of shellac remain dissolved in the chloroform; any color returning after a half minute or so is disregarded.

A blank determination should be run with $20 \mathrm{cc}$. of Wijs solution, 20 cc. of acetic acid, Io cc. of chloroform, and Io cc. of Io per cent. potassium iodide solution. The blank is necessary on account of the well 
known effect of temperature changes on the volume, and possible loss of strength of the Wijs solution.

In the case of grossly adulterated samples or in the testing of pure rosin, it is necessary to use, instead of the $0.2 \mathrm{~g}$. of material, a smaller amount, say 0.15g. or even O.I g., in order that the excess of iodine monochloride may not be too greatly reduced, as the excess of halogen is one of the factors in determining the amount of absorption. It is safe to say that in case less than $25 \mathrm{cc}$. of thiosulphate solution are required, another test should be made, using a smaller amount of the shellac to be tested.

In weighing shellac. some difficulty is at times experienced on account of its electrical properties; in very dry weather it may be found that the necessary handling to prepare it for weighing has electrified it and that it may be necessary to leave it on the balance pan at rest for a few minutes before taking the final weight.

In any halogen absorption process it is essential that the substance to be examined should be thoroughly dissolved in a reasonable volume of solvent that is cheap and easily obtainable. In this case glacial acetic acid forms an admirable solvent; the only other solvents which are at ail comparable with it are the alcohols, which are too readily attacked by halogens to be used with perfect safety.

The two defects which we have found in glacial acetic acid as a solvent are, first, that it solidifies at a temperature but little below that of the ordinary room, and, second, that the results obtained are to a certain extent dependent upon the strength of the acid used. On account of the first mentioned property it may happen that different lots of glacial acelic acid or of Wijs solution may not be of uniform composition throughout the bottle on account of having been frozen and remelted; this difficulty can easily be obviated by remembering to shake the bottles of acid and of Wijs solution before using the contents; to a neglect of this precalution we are disposed to attribute irregular results which we have sometimes obtained during cold weather.

The second difficulty is entirely obviated by using at all times acid of the same standard strength. Three prominent methods by which the strength of the acetic acid might be determined, namely, titration with alkali, the determination of specific gravity, and the determination of the melting point, we have investigated as to their suitability for use in this connection. Of the three we greatly prefer the melting point determination, both on the score of accuracy and of speed. Titration we find least accurate of all, and such accurate determinations of specific gravity as would be necessary in making use of it as a criterion of purity would require an almost prohibitive amount of time and attention. Furthermore, the ultimate standard of a melting point determination, an accurate ther- 
mometer, is a much more satisfactory one and one more easily duplicated than is the case in the other two nethods.

We have, therefore, in the description of the method, specified rather the melting point of the acid to be used than its strength as expressed by percentage.

The metinod which we have used is a simple and rapid one, heing in fact the "titre" method in common use for determining the melting point of fatty acids generally. An s-inch test tube is about half filled with the acid to be tested, the contents chilled rapidy to about $10^{\circ}$ or $I^{\circ}$ and a delicate and accurate themometer, graciuated at least to tenths of a degree, suspended in the acid in the test tube, the latter being placed in a wider test tube closed by a cork through which the test thbe containing the acid is inserted; the whole arrangement is chilled by being immersed in ice water; the acetic acid in a few ninutes begins to crystallize, and the temperature of the supercooled acid rises to its true melting point, where it remains stationary for some time. In the case of acetic acid of such strength as is used in the process this simple procedure gives the required information in a few minutes; with weaker acetic acid greater care is necessary to insure accuracy. The acid in the test tube shond not have begun to crystalize until some ninutes after it has been chilled in cold water and mounted in a larger tube; if it does begin to crystallize too soon, that is, before the cooled acid has had sufficient time to become of uniforn temperature throughout, the acid should be well stirred with the thermometer before the crystallizing process has gone very far.

The melting point of acetic acid containing various percentages of water has been determined by Rudorff, as follows:

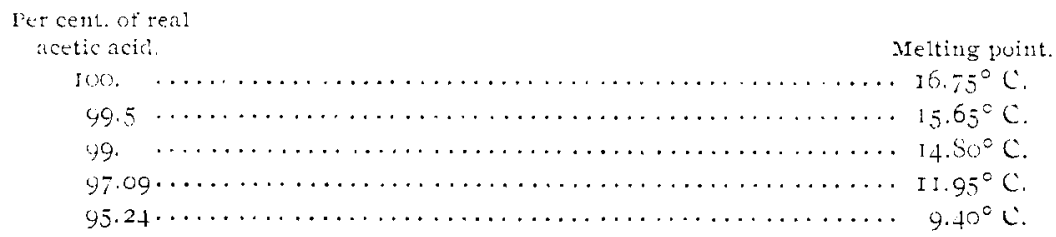

In Thorpe's Dictionary of Applied Chemistry and also in Rudorff's paper in the Pharmaceutical Journal and Transactions the melting point given for acid of 99.5 per cent. strength is $16.65^{\circ}$, but this appears to have been a misprint.

A great deal of work had been done with this process before it was understood that the strength of the acid had any serious effect upon the results; numerous lots of acid had been obtained and used which as the event proved must all have been of approximately the same strength; by

I Berichte, 3, 390, and Phamacentical Journal and I'rensactions, [3] 2, 24 I. Sec also Beilstein, 3d Fd, 1, 399-400. 
comparing the results previously obtained upon standard sanples of shellac, using acid of untested strength, with those later obtained with acids of known melting point, it appears that the mass of valuable data which had been obtained, largely by Dr. Langmuir, concerning the iodine figures of pure shellac had been obtained with acid having a melting point of approximately $14.8^{\circ}$. We have, therefore, adopted as the standard strength of the acid used in this process the strength which corresponds to a melting point of $14.7^{\circ}$ to $15^{\circ}$.

In order to ascertain quantitatively what are the effects of using acids of different strength, we have made several series of experiments in which the strength of the acid was the only variable. One such series was made upon a lot of shellac which had previously been tested a great many times and had always given a figure of approximately $17.7^{\circ}$. A quantity of Wijs solution was prepared, using acid having a melting point of I $4.95^{\circ}$, and with this Wijs solution three tests were made upon the same shellac, using in each test for dissolving the shellac acetic acid of different strength; acid of II. $80^{\circ} \mathrm{M}$. P. gave an iodine figure of I4.67, acid of $14.95^{\circ} \mathrm{M}$. P. (the same as that used for the preparation of the Wijs solution) gave an iodine figure of $I 7.59$, while acid of $I 6.40^{\circ} \mathrm{M}$. P. gave an iodine figure of 20.48 .

We have satisfied ourselves that equally correct determinations of the proportion of shellac and rosin in a mixture can be made by the $\mathrm{Hübl}$, the Hanus, the Wijs and the bromine methods, providing that in each case pains are taken to examine a considerable number of pure shellacs and of rosins to form a satisfactory basis of calculation.

Of these methods the bromine method is the simplest, as the standard solution is very simply made by adding a given volume of bromine to a given volume of glacial acetic acid.

The Hanus solution comes next in simplicity, but we are of the opinion that if sufficient care be taken in the preparation of the iodine bromide solution to insure the exact relation of iodine and bromine to each other that is necessary, there is little to choose in this respect between the Hanus and Wijs methods.

The reliability of any of these methods depends largely upon the number of determinations which have been made by it upon samples of shellac and of rosin which are known to be pure. In this respect we believe the Wijs method to be very decidedly preferable, on account of the number of data obtained by Dr. Langmuir by this method, and using the particular conditions advocated in his paper already referred to. We have carefully considered the merits of each of these conditions, the weight, volume, time, and temperature, and are of the opinion that they can not for general use be modified with advantage. 
Tubleached shellac, when examined by this proces, shows an jodine absoriotion varying from is to is per cent.

No pure shellacs show a higher percentage than $\mathrm{r}$. As shellac is relatively a high priced material and an the variation between its highest and lowest figure is not great. We beliere that sis should be taken an the stanclard figure for shellac deternind by the nethod which we have described.

In the case of rosin the variation between the highest and the lowest figures is much greater; different grades of rosin give figures which vary from about 175 in the darkest varieties to about 260 in the rery lightest colored or W. IT. grade. Of conse theoretically, any gracle of rosin might be mixerl with shellac, but the grades that are actually ued in shellac adulteration seem to be intermediate ones. having probable iodine figures, as determined by this method, between 200 and 225 .

As it is an accepted principle that a stanclard method should lo. so devised that it inaccuracies shall work in tha direction of faroring the seller rather than of condemning tor sererely the article sold, we approve the rather high value taken by I)r. Langmuir for rosili, 22s. aithough if the shellac is dark in color the rosin used to adulterate it may also be datk and have a somewhat lower iodine figure. The results of 11 sing in this method the figure is as the iodine figure of shellac and $22 \mathrm{~s}$ an the figure for rosin may be, under some circumstances, that a slightly lower percentage of rosin will be found than that actually present.

$$
\begin{array}{cccc}
\text { If } y & \text { per cent. rosin } & \\
\text { AI } & \text { iodine number of shellac } \\
N & . " & . & \text { " rosin } \\
\text { A } & \text { ". } & \text { " mixture }
\end{array}
$$

$$
\text { Then } y \quad \text { roo } \frac{A-M}{N-M}
$$

In order to test practically the degree of accuracy and of regularity to be expected from the method, we caused to be prepared by Dr. Langmuir three samples of artificial mixtures of shellac and rosin, designated respectively as "A," containing 5 per cent. of rosin: " $B$," containing 9 per cent., and "C." containing in. 5 per cent. These samples were dis. tributed to the nembers of the committee and analyzed, the true composition remaining unknown to those making the test until the work had been completed. Two numbers analyzed the samples by the method here recommended and two others by the Hubl method. The results were as follows:

$\begin{array}{cl}\text { Anayst. } & \text { Metrod } \\ 1 & \text { Committee } \\ \text { I } & \text { Hiibl } \\ 2 & \text { Committee } \\ 3 & \text { Hiib! }\end{array}$

$\begin{array}{cc}\text { Sample "A" } & \text { sample "B" } \\ \text { percent. rosin. } & \text { Der cert. rosin } \\ 3.3 & 7.5 \\ 3.15 & 7.65 \\ 7.2 & 7.15 \\ 4.3 & 7.5\end{array}$


The use of the figure 200 instead of 228 as the iodine figure of rosin by his method will result in giving the true percentage of rosin with a little rreater accuracy in most cases; we believe, however, that in order to zuard against the rather remote possibility that the use of the figure 200 night give a percentage of rosin slightly too high, it is better policy to we the figure $22 s$.

\section{IHE ACTION OF BARIUM PEROXIDE AND HYDROGEN PEROXIDE UPON FORMALDEHYDE.}

By C. Allax Lyford.

Receivec June $: 3,190 \%$.

The problem of the action of peroxides upon solutions of formaldehyde has been left in a rather unsettled condition. The present research has for its purpose the clearing up of this question. While experimenting upon the oxidation of formaldehyde it was believed that by using bariun peroxide there might result mutual oxidation and reduction with the production of barium formate. Upon looking up the literature of the subject the writer found that Geisow ${ }^{1}$ believes to have obtained only barium carbonate and hydrogen from the interaction of these two substances. He say's he was unable to detect formic acid as an intermediate product either in neutral or acid solution. Geison gives the following reaction:

$$
\mathrm{HCHO}+\mathrm{BaO}_{2}=\mathrm{BaCO}_{3}=\mathrm{H}_{2} \text {. }
$$

So far as the writer can ascertain, this work has neither been confirmed nor disproved by other writers.

For a number of reasons this reaction does not seem probable. In the first place, acetaldehyde forms a hard resin with barium peroxide, no methane being evolved. If there were such an easy direct removal of the carbonyl group one would not expect a resin to be formed. Geisow states that the dioxides of lead and manganese acting upon formaldehyde in neutral solution produce formates and hydrogen. It is known that uranium peroxicie acting upon formaldehyde in neutral solution produces uranyl formate and hydrogen.

$$
\begin{aligned}
& \mathrm{PbO}_{2}+2 \mathrm{HCHO}=(\mathrm{HCOO})_{2} \mathrm{~Pb}-\mathrm{H}_{2} . \\
& \mathrm{CO}_{4}+2 \mathrm{HCHO}=(\mathrm{HCOO})_{2} \mathrm{UO}_{2}+\mathrm{H}_{2} .
\end{aligned}
$$

In alkaline solutions it has been shown that hydrogen peroxide acts upon formaldehyde according to the following equation ${ }^{2}$ :

$$
2 \mathrm{HCHO}+2 \mathrm{NaOH}+\mathrm{H}_{2} \mathrm{O}_{2}=2 \mathrm{HCOONa}+\mathrm{H}_{2}+2 \mathrm{H}_{2} \mathrm{O} \text {, }
$$

(in cold), from which it would follow that the reaction with sodium peroxide and sufficiently dilute formaldehyde would be:

$$
2 \mathrm{HCHO}+\mathrm{Na}_{2} \mathrm{O}_{2}=2 \mathrm{HCOONa}+\mathrm{H}_{2} \text {. }
$$

If 30 per cent. formalin be dropped upon sodium peroxide, there is,

1 Geisow, Ber., 37, 515 .

2 Blank and Finkenbeiner, Ber., 31, 2979. 